\title{
Elective orthopaedic surgery and COVID-19: knowledge and perceptions of patients in a global pandemic
}

\author{
Sean Wei Loong $\underline{\mathrm{Ho}}^{1}$, MBBS, FRCS, Tong Leng $\underline{\operatorname{Tan}}{ }^{1}$, MBBS, FRCS, Keng Thiam $\underline{\operatorname{Le}}{ }^{1,2}$, MBBS, FRCS
}

\section{INTRODUCTION}

The COVID-19 pandemic originated in Wuhan, China, in December 2019. ${ }^{(1)}$ It has since developed into a global pandemic, with a total of 2,475,723 cases reported globally and 169,151 confirmed deaths as of 23 April 2020.(2) The effects of this global pandemic are far-reaching, and the challenges faced by orthopaedic surgery globally amid this pandemic have been well described. ${ }^{(3-6)}$ One of the most important considerations during the pandemic has been to reduce transmission risk to both the patient and the surgeon. As such, surgical organisations such as the American Academy of Orthopaedic Surgeons and American College of Surgeons have recommended that elective orthopaedic surgeries be postponed. ${ }^{(4,7)}$ In addition, there has been a significant drain on surgical and protective equipment as well as personnel, with some orthopaedic surgeons redeployed to screening centres. ${ }^{(8)}$

Singapore identified its first case of COVID-19 on 23 January 2020 . $^{(9)}$ The government response to this now global pandemic has been guided by the Disease Outbreak Response System Condition (DORSCON). ${ }^{(10)}$ First formulated after the experience with severe acute respiratory syndrome (SARS), the DORSCON system is used to describe features of the disease outbreak and provide corresponding guidelines on the level of measures that may be required. On 7 February 2020, in view of rising COVID-19 cases, DORSCON Orange was implemented in Singapore to reflect a growing severity. ${ }^{(11)}$ In line with this, our institution's orthopaedic surgeons were advised to reduce any new listings for elective orthopaedic surgery during this time. On 7 April, to further contain COVID-19, a nationwide embargo on elective surgery for stable sports conditions and joint replacement surgeries was implemented.

There is some existing literature on the knowledge and attitudes of physicians regarding COVID-19. ${ }^{(6,12-14)}$ Likewise, there have also been large-scale cross-sectional studies assessing levels of knowledge and perceptions of the public toward COVID-19. ${ }^{(15-17)}$ Most of these studies have found a generally high understanding of COVID-19 among both medical personnel and the general population. However, there is a paucity of information regarding the knowledge and attitudes of patients who have been scheduled for elective orthopaedic surgery during the COVID-19 pandemic. This is important information, considering that as the COVID-19 pandemic situation improves, there is likely to be a graduated return to performing elective orthopaedic surgeries. Understanding patients' perceptions will provide better insight on how to manage expectations as well as plan logistics during the surgical backlog.

In Singapore, the DORSCON structure guided a graduated response regarding elective surgery. While some institutions chose to completely cease elective surgeries, in our institution, elective orthopaedic surgeries were permitted up to 3 April. This provided a unique perspective for patients who were undergoing elective surgeries and gave us an opportunity to investigate patients' knowledge and attitudes towards elective orthopaedic surgery amid a global pandemic. To our knowledge, no study has explored patients' knowledge and attitudes towards undergoing elective orthopaedic surgery during the COVID-19 pandemic.

This study aimed to assess the knowledge of COVID-19 and attitudes towards elective surgery in a cohort of orthopaedic patients who were planned for elective surgery. The secondary aim was to determine whether there are differences in demographics, knowledge of COVID-19, attitudes towards COVID-19 and health risk-taking attitudes between patients who opted to proceed with surgery and those who chose to cancel their surgery.

\section{METHODS}

This was a questionnaire-based cohort study. The study group consisted of consecutive orthopaedic patients (under the care of the three authors) who were planned for elective surgery from 7 February 2020 to 1 April 2020. All patients who were scheduled to undergo elective surgery (i.e. joint arthroplasty and sports surgery procedures) during the study period were included. Patients who were due for emergency or urgent surgery and those whose surgery was cancelled owing to medical reasons were excluded. Ethics approval from the institutional review board was obtained for the study.

An anonymous questionnaire was administered to the patients at a single tertiary institution; patients were informed that the questionnaire was anonymous and voluntary. The questionnaire was completed within the outpatient clinic setting for those who were attending their outpatient clinic appointment, and via telephone for patients who were not in attendance.

The aim of the questionnaire was to assess patient demographics, level of objective knowledge of COVID-19, subjective assessment of COVID-19 severity, subjective assessment of the severity of the patients' own orthopaedic condition, as well as their health risk attitude. The questionnaire was designed by all the authors, and a consolidated list of questions was chosen by consensus. The questionnaire was tested on a sample pool of

${ }^{1}$ Department of Orthopaedic Surgery, Tan Tock Seng Hospital, ${ }^{2}$ Yong Loo Lin School of Medicine, National University of Singapore, Singapore

Correspondence: Dr Sean Wei Loong Ho, Consultant, Department of Orthopaedic Surgery, Tan Tock Seng Hospital, 11 Jalan Tan Tock Seng, Singapore 308433. sean_wl_ho@ttsh.com.sg 
patients to determine the ease of understanding. Revisions were made before the final questionnaire was administered.

The patient demographics that were assessed included age, gender, race, occupation, highest level of education, total household income and whether the respondent or family member had ever worked in healthcare. Respondents were asked a series of questions designed to assess their attitudes towards the severity of COVID-19 and the adequacy of the government response, scored on a five-point scale ( $1=$ agree to a large extent, 2 = agree, 3 = neutral, 4 = disagree, 5 = disagree to a large extent). Respondents were then asked questions to assess their level of knowledge of COVID-19. These questions determined whether the respondents were aware of the symptoms, population at risk and mortality rate of COVID-19. The next segment of questions assessed how respondents viewed the severity and/or urgency of their own condition. An open-ended question was also posed to respondents regarding their reasons for proceeding or cancelling their elective orthopaedic surgery. Finally, the patients completed the Health-Risk Attitude Scale (HRAS), ${ }^{(18)}$ a validated scale that assesses an individual's attitude towards risk, specifically when dealing with matters of health. We chose this instrument over others such as the Domain-Specific Risk-Taking (DOSPERT) scale because of its ease of administration (13 questions vs. 35 questions for DOSPERT) and the fact that the HRAS has been shown to be internally consistent (Cronbach alpha $=0.83$ ) and reliable (test-retest intraclass correlation coefficient $=0.86$ ), with good construct and convergent validity. ${ }^{(19)}$ This instrument consists of 13 questions scored from 1 (agree) to 7 (disagree), with a total score ranging from 13 to 91 . A higher score reflects a more risk-averse attitude.

Descriptive statistics were calculated for the demographics and background variables. Univariate comparisons of means were conducted using Student's $t$-test. Chi-square test was used for comparison of proportions between the two groups. Differences were considered significant when p-values were below 0.05. All analyses were performed in Stata version 15.0 (Stata Corp, College Station, TX, USA).

\section{RESULTS}

A total of 34 patients met the inclusion criteria. Of these, 30 (88\%) completed the questionnaire. The median patient age was 51 (18-82) years, and 19 (63\%) of the 30 patients were male. The total monthly household income of $15(50 \%)$ patients was below the lower quartile, as per the national census. ${ }^{(20)} 5(17 \%)$ patients had a family member who was a healthcare worker. Of the 30 patients, $9(30 \%)$ chose to cancel their surgery and $21(70 \%)$ opted to proceed. No significant differences were observed in demographics such as age, gender, education level or household income between patients who chose to cancel their surgery and those who opted to proceed (Table I).

In terms of perceptions of COVID-19, 24 (80\%) patients thought that it was very easy or easy for a normal individual to get infected with COVID-19. 14 (47\%) patients thought that transmission of COVID-19 within the hospital was very likely or likely, while 16 (53\%) felt worried about contracting COVID-19.
Table I. Demographic information of patients $(n=30)$

\begin{tabular}{|llll|}
\hline Demographic & \multicolumn{2}{c}{ Mean \pm SD/No. (\%) } & p-value \\
\cline { 2 - 3 } & $\begin{array}{l}\text { Proceeded with } \\
\text { surgery }(\mathbf{n}=\mathbf{2 1})\end{array}$ & $\begin{array}{l}\text { Cancelled } \\
\text { surgery (n=9) }\end{array}$ & \\
\hline Age (yr) & $45.6 \pm 21.6$ & $51.8 \pm 21.1$ & 0.48 \\
\hline Gender & $12(57)$ & $7(78)$ & 0.28 \\
\hline \multicolumn{1}{|l}{ Male } & $9(43)$ & $2(22)$ & 0.80 \\
\hline $\begin{array}{l}\text { Female } \\
\text { Highest education } \\
\text { level achieved* }\end{array}$ & $8(38)$ & $3(33)$ & 0.69 \\
\hline $\begin{array}{l}\text { Household } \\
\text { income }\end{array}$ & $10(48)$ & $5(56)$ & 0.59 \\
\hline $\begin{array}{l}\text { Healthcare worker } \\
\text { in the family }\end{array}$ & $3(14)$ & $2(22)$ & \\
\hline
\end{tabular}

*Above high school. †Above lower quartile. SD: standard deviation

Although the majority $(87 \%, \mathrm{n}=26)$ considered COVID-19 to be a national threat, most patients $(87 \%, \mathrm{n}=26)$ felt that the government was handling the situation well.

In terms of knowledge of COVID-19 infection, all the patients were able to identify the at-risk groups (such as the elderly and those with underlying comorbidities) that are more susceptible to infection. $22(73 \%)$ patients were able to correctly select the symptoms associated with COVID-19 infection. Patients with poorer knowledge tended to be less worried about contracting COVID-19 ( $p=0.06$ ). There was no significant effect of socioeconomic status or education level on the degree of knowledge of COVID-19. The presence of a healthcare worker in the family did not significantly improve the degree of knowledge of COVID-19, nor did it affect patients' perception of the severity of COVID-19.

When assessing the severity of their own orthopaedic condition, 11 (37\%) patients felt that surgery for their condition was urgent. Only $1(3 \%)$ patient felt that surgery was risky, although $12(40 \%)$ patients felt that their risk of surgery was elevated owing to the ongoing COVID-19 pandemic. Almost all $(93 \%, n=28)$ of the patients thought that the hospital would either postpone or cancel their surgery if there was an increased risk. When asked why they chose to proceed or cancel their surgery, some common themes surfaced. As expected, the majority $(7 / 9,78 \%)$ who cancelled their surgery cited the primary reason as "worried about the risk of COVID-19" (Table II). For patients who opted to proceed, the reasons included: "faith in the hospital system", "felt that the condition was getting worse" and "COVID-19 situation is not so serious". We found no significant difference in the level of concern about the COVID-19 situation between patients who proceeded and those who cancelled the surgery $(p=0.15)$. However, patients who thought that the hospital was a likely place of transmission were more likely to cancel their surgery $(p=0.07)$. In addition, patients who felt that their surgery was urgent were more likely to proceed with it $(p<0.01)$. The HRAS revealed that patients who proceeded with surgery were less risk averse (mean score $33.5 \pm 6.3$ ) as compared to patients who opted to cancel their surgery (mean 
Table II. Reasons for surgery decision in orthopaedic patients $(n=30)$.

\begin{tabular}{|c|c|c|c|c|c|}
\hline No. & Age (yr) & Gender & Type of surgery & Decision & Reason \\
\hline 1 & 65 & Female & Rotator cuff repair & Proceed & Did not think COVID-19 was serious. \\
\hline 2 & 21 & Male & $\mathrm{ACL}$ reconstruction & Proceed & Wanted to perform surgery during conscription. \\
\hline 3 & 64 & Male & Rotator cuff repair & Proceed & Pain was intolerable. \\
\hline 4 & 21 & Male & $\mathrm{ACL}$ reconstruction & Proceed & $\begin{array}{l}\text { Felt condition was worsening. Felt that he would bother the surgeon if he } \\
\text { postponed surgery. }\end{array}$ \\
\hline 5 & 33 & Male & $\mathrm{ACL}$ reconstruction & Proceed & Felt that surgery was important. \\
\hline 6 & 27 & Male & $\mathrm{ACL}$ reconstruction & Proceed & Did not think COVID-19 was serious. \\
\hline 7 & 20 & Male & $\mathrm{ACL}$ reconstruction & Proceed & Wanted to get back to full function as soon as possible. \\
\hline 8 & 21 & Male & MPFL reconstruction & Proceed & Wanted to perform surgery during conscription. \\
\hline 9 & 22 & Male & $\mathrm{ACL}$ reconstruction & Proceed & Did not think COVID-19 was serious. \\
\hline 10 & 29 & Male & Bankart repair & Proceed & $\begin{array}{l}\text { Did not think COVID-19 was serious. Had faith that hospital would take } \\
\text { precautions. }\end{array}$ \\
\hline 11 & 18 & Male & $\mathrm{ACL}$ reconstruction & Proceed & Wanted to get back to full function as soon as possible. \\
\hline 12 & 52 & Male & Rotator cuff repair & Proceed & $\begin{array}{l}\text { Had faith in the institution, and thought they would cancel the surgery if it was } \\
\text { not safe to proceed. }\end{array}$ \\
\hline 13 & 68 & Female & TKA & Proceed & Pain was intolerable. \\
\hline 14 & 73 & Female & TKA & Proceed & Pain was intolerable. \\
\hline 15 & 50 & Male & TKA & Proceed & $\begin{array}{l}\text { Pain was intolerable. Had faith in the institution, and thought they would cancel } \\
\text { the surgery if it was not safe to proceed. }\end{array}$ \\
\hline 16 & 56 & Female & UKA & Proceed & Poor quality of life, pain was worsening. \\
\hline 17 & 74 & Female & TKA & Proceed & $\begin{array}{l}\text { Had faith in the institution, and thought they would cancel the surgery if it was } \\
\text { not safe to proceed. }\end{array}$ \\
\hline 18 & 68 & Female & UKA & Proceed & Felt her condition was getting worse. \\
\hline 19 & 67 & Female & TKA & Proceed & Felt her condition was getting worse. \\
\hline 20 & 73 & Female & TKA & Proceed & Had faith in the hospital and religious beliefs. \\
\hline 21 & 37 & Female & Rotator cuff repair & Proceed & $\begin{array}{l}\text { Had faith in the hospital, and thought they would cancel the surgery if it was } \\
\text { not safe to proceed. }\end{array}$ \\
\hline 22 & 72 & Female & TKA & Cancel & Worried about risk of COVID-19. \\
\hline 23 & 64 & Male & TKA & Cancel & Worried about risk of COVID-19. \\
\hline 24 & 82 & Male & TKA & Cancel & $\begin{array}{l}\text { Felt transportation and follow-up would be difficult for family during COVID-19 } \\
\text { pandemic. }\end{array}$ \\
\hline 25 & 58 & Male & Rotator cuff repair & Cancel & Worried about risk of COVID-19. \\
\hline 26 & 21 & Male & $\mathrm{ACL}$ reconstruction & Cancel & Worried about risk of COVID-19. \\
\hline 27 & 29 & Male & $A C L$ reconstruction & Cancel & $\begin{array}{l}\text { Worried about risk of COVID-19. Did not want to infect the surgical team if he } \\
\text { were an asymptomatic carrier. }\end{array}$ \\
\hline 28 & 46 & Male & Meniscus procedures & Cancel & Worried about risk of COVID-19. \\
\hline 29 & 63 & Female & Meniscus procedures & Cancel & Felt surgery was not urgent. \\
\hline 30 & 31 & Male & $A C L$ reconstruction & Cancel & $\begin{array}{l}\text { Worried about risk of COVID-19. Did not want to infect the surgical team if he } \\
\text { were an asymptomatic carrier. }\end{array}$ \\
\hline
\end{tabular}

ACL: anterior cruciate ligament; MPFL: medial patello-femoral ligament; TKA: total knee arthroplasty; UKA: unicompartmental knee arthroplasty

score $37.2 \pm 7.2)$, although the difference did not reach statistical significance $(p=0.17)$.

\section{DISCUSSION}

COVID-19 is a global pandemic that has significantly affected healthcare systems. Its ripple effects on orthopaedic surgery are substantial. In particular, elective surgery has suffered over the last few months while healthcare systems were repositioning to better tackle the demands of the pandemic. The rationale for a complete embargo on elective surgery includes reducing unnecessary risks for both the patient and surgeon, as well as conservation of drugs and medical equipment. ${ }^{(5,8)}$ However, there are also advantages to proceeding with previously listed electives. In addition to alleviating pain and disability in patients, it also reduces the likelihood of a mounting backlog of surgeries after the pandemic resolves. Any decision to proceed with elective surgery during a pandemic should be made relative to the disease curve and with consideration of hospital resources. In select healthcare systems that choose to proceed with elective surgery, it is important to understand the patients' perceptions and knowledge of COVID-19, as it allows healthcare systems to better counsel patients and plan for their surgery. At the National Centre for Infectious Diseases, duties were split between staffing reserved for COVID-19 and maintaining some degree of 'business 
as usual'. During the study period, our orthopaedic surgery department continued to clear previously listed electives and stopped all new listings. As of 3 April 2020, a complete embargo on elective surgeries was imposed.

Our study revealed that knowledge of COVID-19 was relatively high among our patients who were planned for elective orthopaedic surgery. This is consistent with other crosssectional studies that have reflected a high level of COVID-19 knowledge among the public. ${ }^{(16,17)}$ Interestingly, we found that traditionally vulnerable groups, such as the elderly ${ }^{(16)}$ and socioeconomically disadvantaged individuals, ${ }^{(16,17)}$ possessed a high level of COVID-19 knowledge. This is likely attributable to the considerable mainstream media coverage of this global pandemic, which also highlights the extensive efforts of the government in public education.

In terms of the patients' view of COVID-19 as a personal threat, we found that the majority $(80 \%)$ thought that it was very easy or easy for a normal individual to get infected. Despite this, only about half $(47 \%)$ of the patients thought that transmission within the hospital would be very likely or likely. This perception affected whether a patient proceeded with surgery or not. Patients who proceeded with surgery were more likely to feel that the hospital was a safe environment, regardless of their concerns about COVID-19. This study also revealed that most patients $(28 / 30,93 \%)$ trusted that the hospital would cancel the surgery if there was an increased risk. This is consistent with the reasons given by patients who proceeded with surgery (i.e. "faith in the hospital system"). It is also important to note that patients who cancelled and those who proceeded with surgery had similar HRAS scores, as well as similar levels of concern about contracting COVID-19. Among patients who cancelled their surgery, the majority $(7 / 9,78 \%)$ cited the main reason as "worried about the risk of COVID-19".

Altogether, these findings suggest that trust in the healthcare system is a key component of decision-making in patients. This information should guide patient counselling in a pandemic environment. Surgeons should be aware that despite concerns, patients who proceed with elective surgery often do so because they trust the hospital system and their surgeon. During a pandemic, the patient may have the expectation that the hospital would cancel the surgery if the risks are deemed to be high. As such, surgeons should be cognisant of this fact and proactively engage the patient in operative risk assessment about COVID-19 as part of preoperative counselling. Appropriate patient counselling regarding COVID-19 operative risk assessment is also likely to reduce the number of potential unnecessary surgical cancellations from patients. This is particularly critical to maximise surgical resources.

Patients who felt that their condition was worsening or severe were more likely to proceed with surgery. As healthcare systems ramp up towards 'business as usual', it is likely that most will face a backlog of surgeries. Perceived severity is a key concern for patients. This issue should be addressed in the event of delays in performing surgery. First, surgeons should consider appropriate patient education that is condition-centric. For example, in stable degenerative arthropathies, patients should be counselled that their surgery can be safely postponed because their condition is unlikely to worsen rapidly, and that similar surgical outcomes can be expected when the surgery is eventually performed. For patients who are in persistent pain, interim procedures such as nerve blocks or intra-articular injectables should be offered to reduce symptomology while awaiting surgery.

It is likely that the COVID-19 pandemic will have continued implications for elective surgery. This study has highlighted that while patients who are planned for elective surgery have high levels of knowledge about COVID-19, it is still prudent to offer counselling, specifically on any additional risks that COVID-19 may confer. In addition, condition-centric patient education, coupled with interim procedures aimed at reducing pain, can address some key concerns of patients with stable degenerative orthopaedic conditions. As we recover from the pandemic, it is important to continuously engage patients. If a patient's key concerns are not well addressed, it is unlikely that the patient will accept continuous delays in elective surgery. Such actions will ultimately erode trust in the patient-doctor relationship.

Our study is not without limitations. First, we accept that the sample size in our study was small and no power analysis was performed owing to the absence of comparable published data. Second, this study was conducted in February and March 2020, when there were relatively low numbers of daily COVID-19 cases and fewer hospitalised COVID-19 patients in Singapore. These factors could have influenced our patients' responses. Finally, we concede that our findings may not be generalisable to other populations. Thus, we recommend that further studies be undertaken to better define patients' perceptions of elective surgery amid a global pandemic.

In conclusion, a high level of knowledge regarding COVID-19 was observed in our cohort of patients undergoing orthopaedic elective surgery. Key reasons for proceeding with surgery amid a pandemic include trust in the hospital system and patients' selfperceived severity of their condition. It is important to address patients' concerns with appropriate patient education and interim procedures when planning for elective orthopaedic surgery during a pandemic.

\section{REFERENCES}

1. Guan WJ, Ni ZY, Hu Y, et al. Clinical characteristics of coronavirus disease 2019 in China. N Engl J Med 2020; 382:1708-20.

2. World Health Organization. Coronavirus disease (COVID-19) pandemic. Available at: https://www.who.int/emergencies/diseases/novelcoronavirus-2019. Accessed April 22, 2020.

3. Lei S, Jiang F, Su W, et al. Clinical characteristics and outcomes of patients undergoing surgeries during the incubation period of COVID-19 infection. EClinicalMedicine 2020; 21:100331.

4. Lacobucci G. COVID-19: all non-urgent elective surgery is suspended for at least three months in England. BMJ 2020; 368:m1106.

5. Stahel PF. How to risk-stratify elective surgery during the COVID-19 pandemic? Patient Saf Surg 2020; 14:8.

6. Angelos P. Surgeons, ethics, and COVID-19: early lessons learned. J Am Coll Surg 2020; 230:1119-20.

7. American Academy of Orthopaedic Surgeons. COVID-19: guidelines for elective surgery. Available at: https://www.aaos.org/about/covid-19-information-for-ourmembers/aaos-guidelines-for-elective-surgery/. Accessed April 22, 2020.

8. Soh TLT, Ho SWL, Yap WMQ, Oh JYL. Spine surgery and COVID-19: challenges and strategies from the front lines. J Bone Joint Surg Am 2020; 102:e56.

9. Wong JEL, Leo YS, Tan CC. COVID-19 in Singapore-current experience: critical 
global issues that require attention and action. JAMA 2020; 323:1243-4.

10. What do the different DORSCON levels mean. In: Government of Singapore [online]. Available at: https://www.gov.sg/article/what-do-the-different-dorsconlevels-mean. Accessed April 22, 2020.

11. Ministry of Health, Singapore. Risk assessment raised to DORSCON orange. Available at: https://www.moh.gov.sg/news-highlights/details/risk-assessmentraised-to-dorscon-orange. Accessed April 22, 2020.

12. Shi Y, Wang J, Yang Y, et al. Knowledge and attitudes of medical staff in Chinese psychiatric hospitals regarding COVID-19. Brain Behav Immun Health 2020; 4:100064.

13. Bhagavathula AS, Aldhaleei WA, Rahmani J, Mahabadi MA, Bandari DK. Novel coronavirus (COVID-19) knowledge and perceptions: a survey of healthcare workers. medRxiv 2020 Mar 16. https://doi.org/10.1101/2020.03.09.2003338 1. Preprint.

14. Moro M, Vigezzi GP, Capraro M, et al. 2019-novel coronavirus survey: knowledge and attitudes of hospital staff of a large Italian teaching hospital. Acta Biomed 2020; 91(3-S):29-34.

15. Geldsetzer P. Knowledge and perceptions of COVID-19 among the general public in the United States and the United Kingdom: a cross-sectional online survey. Ann Intern Med 2020; 173:157-60

16. Abdelhafiz AS, Mohammed Z, Ibrahim ME, et al. Knowledge, perceptions and attitude of Egyptians towards the novel coronavirus disease (COVID-19). J Community Health 2020; 45:881-90.

17. Zhong BL, Luo W, Li HM, et al. Knowledge, attitudes, and practices towards COVID-19 among Chinese residents during the rapid rise period of the COVID-19 outbreak: a quick online cross-sectional survey. Int J Biol Sci 2020; $16: 174$

18. van Osch SMC, Stiggelbout AM. The development of the Health-Risk Attitude Scale. Leiden: Leiden University Repository. Available at: https://openaccess. leidenuniv.nl/bitstream/handle/1887/12363/07.pdf?sequence=10. Accessed April 20, 2020

19. Bansback N, Harrison M, Sadatsafavi M, Stiggelbout A, Whitehurst DGT. Attitude to health risk in the Canadian population: a cross-sectional survey. CMAJ Open 2016; 4:E284-91.

20. Department of Statistics, Singapore. Available at: https://www.tablebuilder. singstat.gov.sg/publicfacing/createDataTable.action?refld=12307. Accessed April 20, 2020. 